\title{
HIGH PREVALENCE OF STAPHYLOCOCCUS AUREUS NASAL CARRIAGE AMONG CHILDREN IN SZOLNOK, HUNGARY
}

\author{
KriszTinA LAUB, AdRIENN TÓTHPÁL, EsZTER KOVÁCS, JUdiT SAHIN-TÓTH, \\ Andrea Horváth, SzILVIa Kardos and OrSOlya DOBAY*
}

Institute of Medical Microbiology, Semmelweis University, Budapest, Hungary

(Received: 13 October 2017; accepted: 8 November 2017)

We collected nasal samples from 1,390 healthy 3-7 years old children in Szolnok city, Hungary, in 2012. We detected 476 Staphylococcus aureus isolates from 474 children. In two occasions, two different $S$. aureus were isolated, based on hemolysis type and pulsed-field gel electrophoresis pattern. S. aureus carriage rate was calculated to be $34.1 \%$ similar to others studies. Male gender was found to be a risk factor for carriage by statistical analysis. Altogether, four methicillin-resistant $S$. aureus (MRSA) strains were detected by mecA polymerase chain reaction, which means $0.8 \%$ community-acquired MRSA prevalence among the $S$. aureus isolates. All MRSA strains harbored the SCCmec type IV cassette (typical for CA-MRSA) and belonged to ST45 by multilocus sequence typing. During antibiotic susceptibility testing, we measured the following resistance rates: $0.0 \%$ for mupirocin, $0.2 \%$ for ciprofloxacin, $0.6 \%$ for gentamicin and oxacillin, $3.4 \%$ for tetracycline, $9.5 \%$ for clindamycin, $10.3 \%$ for erythromycin, and $91.4 \%$ for penicillin, which are generally lower compared with Hungarian clinical isolates.

Keywords: Staphylococcus aureus, CA-MRSA, carriage, children, antibioticresistance, Hungary

\section{Introduction}

Staphylococcus aureus is one of the most important pyogenic bacteria causing infections in the community and hospitals as well. It can colonize the skin and mucosal surfaces of humans and several animal species also. The anterior nares of the nose are the most frequent carriage sites for this bacterium; however, extranasal sites can harbor S. aureus, for example, skin, perineum, pharynx, gastrointestinal tract, vagina, and axillae. Based on longitudinal studies, carriers

\footnotetext{
*Corresponding author; E-mail: dobay.orsolya@med.semmelweis-univ.hu
} 
can be distinguished into persistent, intermittent, and non-carriers. Approximately $20 \%$ of humans are persistent $S$. aureus nasal carriers, whereas $30 \%$ are intermittent carriers. Children are more commonly persistent carriers than adults. For instance, more than $70 \%$ of newborn babies' nasal samples are positive for $S$. aureus at least once. The association between staphylococcal disease and its nasal carriage was first declared in 1931 by Danbolt studying furunculosis. Persistent carriers have a higher risk of getting staphylococcal infection [1]. Wertheim et al. [1] have defined several risk factors for $S$. aureus nasal carriage in a review, such as ethnicity (white people have higher rates), male gender, patients with diabetes mellitus, hemodialyzed or continuous peritoneal-dialyzed patients, patients having end stage liver disease, HIV infection, obesity, previous cerebrovascular accident, S. aureus skin infections, chronic skin disease (like eczema, atopic dermatitis, or psoriasis), people doing contact sport or other activities leading to skin lesions, as well as animal farming.

Methicillin-resistant $S$. aureus (MRSA) strains were first reported from England by Barber [2]. Within two decades, health care-associated MRSA (HA-MRSA) strains spread all over the USA causing severe invasive infections with high mortality [3]. In the mid-1990s, community-associated MRSA (CA-MRSA) was described and since that it spread worldwide. It can be distinguished from HA-MRSA strains by genetic patterns and antibiotic resistance also. Community-acquired MRSA strains mainly harbor staphylococcal cassette chromosome (SCCmec) types IV, V, or VII, whereas most hospital-acquired MRSA harbors SCCmec types II and III. CA-MRSA is usually more susceptible to non- $\beta$-lactam antibiotics compared with HA-MRSA. Another molecular typing method, multilocus sequence typing (MLST) is also commonly used to demonstrate different $S$. aureus clones and their global spread. For instance, CA-MRSA strains in the US most frequently belong to USA300:ST8 and USA400:ST1 clones, whereas in Europe, ST80 strains predominate [4].

The aim of this study was to estimate the $S$. aureus nasal carriage rate in the healthy young children population in a given Hungarian city (Szolnok) and to characterize the strains using molecular techniques as well. We also determined the antibiotic resistance rates of the strains to several groups of commonly used antibiotics.

\section{Materials and Methods}

\section{Study population}

We collected nasal samples from 1,390 healthy 3- to 7-year-old children in 20 different day-care centers (DCCs) in Szolnok, Hungary, between February and 
June 2012. Szolnok is one of the county towns of Hungary and its population was about 73,000 inhabitants during the study. Before the sample collection, the parents gave their informed consent for the process and filled out a questionnaire specialized for possible risk factors for carriage, like gender, having siblings, whether the siblings attended any community, and finally passive smoking. Unfortunately, they did not always answer all the questions, which caused the differences in the total number of children during the analysis of risk factors. We applied $\chi^{2}$ test for statistical analysis, where appropriate.

\section{Sample collection}

We used soft cotton swabs for sample collection, which was taken from both nostrils. Then, the swabs were inserted into active charcoal containing Amies transport media (Transwab, Medical Wire \& Equipment, Corsham, UK) and transported to the microbiology laboratory within $24 \mathrm{~h}$.

\section{Phenotypical identification}

The nasal samples were inoculated onto Mueller-Hinton blood agar plates and after an overnight incubation at $37{ }^{\circ} \mathrm{C}$, in $5 \% \mathrm{CO}_{2}$, the $S$. aureus suspicious $\beta$-hemolytic colonies were isolated to produce pure culture. First, we confirmed the identity of $S$. aureus isolates by catalase test and clump test (Pastorex StaphPlus Kit, Bio-Rad, Marnes-la-Coquette, France) and every S. aureus suspicious isolate was frozen and kept at $-80{ }^{\circ} \mathrm{C}$ on cryobeads (Cryobank, Mast Diagnostica, Bootle, UK) until further examinations.

\section{Genotypical identification by polymerase chain reaction (PCR)}

For further genetic identification of the isolates, we detected the $S$. aureus species-specific thermonuclease gene by nucA PCR. For MRSA screening, we applied mecA PCR for all isolates. The nucA and mecA gene amplifications were combined into one multiplex PCR reaction. We designed both primer pairs in our laboratory as described before [5]. For the PCR, $2 \frac{1}{2} \mu 1$ of boiled bacterium templates $\left(99^{\circ} \mathrm{C}, 15 \mathrm{~min}\right.$ ) was prepared. We applied the following cycling parameters: $3 \mathrm{~min}$ at $94^{\circ} \mathrm{C}$, then 30 cycles of $94^{\circ} \mathrm{C}$ for $60 \mathrm{~s}$, $54^{\circ} \mathrm{C}$ for $60 \mathrm{~s}, 72^{\circ} \mathrm{C}$ for $30 \mathrm{~s}$, and a final extension of $72^{\circ} \mathrm{C}$ for $10 \mathrm{~min}$. Each reaction included ATCC 33591 as a $n u c A$, mecA positive external control strain. 
For more correct MRSA screening, we checked mecC gene also in isolates with oxacillin minimum inhibitory concentration (MIC) $\geq 1 \mathrm{mg} / \mathrm{ml}$ by PCR in a separate reaction. It was done after Paterson et al. [6] using ATCC BAA-2312 as a positive control strain.

\section{SCCmec typing}

SCCmec typing was performed in the case of the four mecA positive isolates found in this study. As CA-MRSA isolates mostly belong to the SCCmec types IV or $\mathrm{V}$, these two types were checked. Parts of the respective type-specific recombinase genes ( $c c r)$ were amplified using the primers designed by Milheiriço et al. [7], and the $\mathrm{J} 3$ region was detected in addition for type IV, with primers published by Oliveira and de Lencastre [8]. The sizes of the PCR products were 311 (ccr-IV), 449 (ccr-V), and 342 (J3-IV).

\section{MLST}

Well-defined sections of seven housekeeping genes ( $\operatorname{arcC}$, aroE, $g l p F, g m k$, pta, tpi, and yqiL) of the four mecA positive isolates were amplified by PCR, using the primers provided on the MLST website [9]. The following cycling parameters were applied: $5 \mathrm{~min}$ at $95^{\circ} \mathrm{C}$, then 30 cycles of $95^{\circ} \mathrm{C}$ for $60 \mathrm{~s}, 55^{\circ} \mathrm{C}$ for $60 \mathrm{~s}, 72^{\circ} \mathrm{C}$ for $60 \mathrm{~s}$, and a final extension of $72^{\circ} \mathrm{C}$ for $5 \mathrm{~min}$. The products were purified by the QIAquick PCR purification kit (Qiagen, Germany) and sent for sequencing to BIOMI Ltd., Gödöllö, Hungary. The allele sequences were compared with the MLST database and the sequence types identified.

\section{Genotyping by pulsed-field gel electrophoresis (PFGE)}

In the case of special $S$. aureus isolates found in this study (co-carried strains as detailed below), we applied PFGE method to determine their genetic relatedness. The same conditions and running parameters were used as described earlier in our previous study [5].

\section{Antibiotic susceptibility testing}

We determined the MIC to eight antibiotics: penicillin, oxacillin, erythromycin, clindamycin, tetracycline, gentamicin, ciprofloxacin, and mupirocin by agar dilution method in Mueller-Hinton agar plates, using an A400 Multipoint 
Inoculator (AQS Manufacturing Ltd., Southwater, UK). In all cases, the European Committee on Antimicrobial Susceptibility Testing (EUCAST) guidelines and breakpoints were applied, and the ATCC 29213 control strain was used. For the more accurate detection of MRSA, cefoxitin discs (30 $\mu \mathrm{g}$, Bio-Rad) were used parallel to oxacillin MICs according to the EUCAST recommendations [10]. To detect inducible clindamycin resistance, double disk diffusion was applied, using $15 \mu \mathrm{g}$ erythromycin and $2 \mu \mathrm{g}$ clindamycin disks (Bio-Rad).

\section{Determination of hemolysis type}

The method described by Herbert et al. [11] was used to compare the hemolytic activity of the two $S$. aureus strains each isolated from the same child (as observed in two instances). Briefly, the isolates in question were cross-streaked perpendicularly to RN4220 (kindly provided by Prof. Richard Novick) and based on the interaction with this test strain, hemolysis type was determined.

\section{Results}

\section{Carriage rate}

In total, we screened 1,390 preschool children (3-7 years old) in Szolnok, Hungary, attending DCCs, including all the 20 existing DCCs in this city between February and June 2012. Gender of the child was unknown in one case; considering this, out of 1,389 children, $52.7 \%(n=732)$ were males and $47.3 \%(n=657)$ were females. We could detect $S$. aureus in 474 cases, which equals to $34.1 \%$ carriage rate. From two samples, two different $S$. aureus were isolated (as detailed below). Hence, a total of $476 \mathrm{~S}$. aureus were identified.

\section{Risk factors}

Analyzing the questionnaires, we focused on possible risk factors of nasal carriage. Out of the 474 carriers, $58.2 \%(n=276)$ were males, whereas among the non-carriers, this ratio was $49.8 \%$, and this difference was found to be statistically significant (Table I). We had information about the sibling status in the case of 470 carriers and $69.1 \%(n=325)$ of them had one or more siblings. Compared with it, a quite similar proportion (70.5\%) was found among the non-carriers, which is statistically not significant. We had data about whether the siblings attended any community or not. Out of 325 carriers, $88.6 \%(n=288)$ of their siblings were 
Table I. Correlation between the analyzed risk factors and Staphylococcus aureus carriage

\begin{tabular}{lccc}
\hline Risk factor & Carrier & Non-carrier & $\chi^{2}$ test \\
\hline Male & $\mathbf{2 7 6}(\mathbf{3 7 . 7 \%})$ & $\mathbf{4 5 6}(\mathbf{6 2 . 3} \%)$ & $\boldsymbol{p}=\mathbf{0 . 0 0 3}$ \\
Female & $198(30.1 \%)$ & $459(69.9 \%)$ & \\
Having siblings & $325(33.7 \%)$ & $639(66.3 \%)$ & NS $(p=0.62)$ \\
Not having siblings & $145(35.1 \%)$ & $268(64.9 \%)$ & \\
Siblings attending community & $288(34.4 \%)$ & $548(65.6 \%)$ & NS $(p=0.34)$ \\
Siblings not attending community & $37(30.1 \%)$ & $86(69.9 \%)$ & \\
Passive smoking & $177(32.7 \%)$ & $364(67.3 \%)$ & NS $(p=0.38)$ \\
No passive smoking & $293(35.0 \%)$ & $544(65.0 \%)$ &
\end{tabular}

Note: Statistically significant risk factors are represented in bold. NS: not significant.

member of some kind of community (DCC or school) in contrast with $86.4 \%$ of the non-carriers' siblings. This is also statistically non-significant. Passive smoking was considered as well, and we calculated $37.7 \%(n=177)$ prevalence among the 470 carriers, whereas $40.1 \%$ among the non-carriers, which again was not statistically significant (Table I).

Antibiotic susceptibility and molecular typing of the MRSAs

Out of the 476 isolates, three proved to be oxacillin- and cefoxitin-resistant. Moreover, we found the mecA gene in one additional strain, which showed phenotypic oxacillin-sensitivity (MIC $=1 \mathrm{mg} / \mathrm{L}$ ) and cefoxitin-sensitivity $(23 \mathrm{~mm}$ zone diameter) as well. The mecA PCR product of this strain was verified by sequencing. Therefore, we altogether identified four MRSAs, from which one was an oxacillin-sensitive strain. According to this, CA-MRSA carriage rate among all examined children was $0.3 \%$, which means a $0.8 \%$ MRSA prevalence among the S. aureus strains. All four MRSA isolates harbored the SCCmec type IV cassette and belonged to sequence type 45 (ST45) by MLST.

Antibiotic susceptibility testing was a main part of this study as we measured MIC values for eight different antibiotics for all isolates. We detected $100 \%$ sensitivity only in the case of mupirocin. For ciprofloxacin and gentamicin, most of the isolates were sensitive ( $99.8 \%$ and $99.4 \%$, respectively). As mentioned above, there were three oxacillin-resistant strains, which resulted in $0.6 \%$ resistance rate. Tetracycline-resistance appeared to be $3.4 \%$, whereas $10.3 \%$ of the isolates were resistant to erythromycin. Clindamycin resistance was calculated to be $9.5 \%$, including inducible resistance, which was $9.0 \%$ per se. The highest resistance $-91.4 \%$ - was measured for penicillin of course. All four MRSA strains showed a high level of erythromycin resistance (MIC $\geq 512 \mathrm{mg} / \mathrm{L}$ ) as well. In addition, two methicillin-susceptible $S$. aureus isolates exhibited multidrug 
resistance (i.e., resistant to three or more different classes of antibiotics): one resistant to penicillin, ciprofloxacin, and erythromycin and one resistant to penicillin, gentamicin, and erythromycin.

\section{Co-carriage of two $\mathrm{S}$. aureus strains by the same child}

In two occasions, two phenotypically different $S$. aureus were isolated from the same nasal sample, based on colony morphology (L265/1-2 and L850/1-2). To determine whether these isolates were identical or different, their antibiotic sensitivity, hemolysis type, and PFGE pattern were compared. Regarding the antibiotic susceptibility, all four isolates had a different penicillin MIC, but their MICs for the other antibiotics were the same (sensitive to all antibiotics tested, except for penicillin), as shown in Table II.

The hemolysis types (Figure $1 \mathrm{a}$ and $\mathrm{b}$ ) as well as the PFGE patterns of the strains were also different in both cases; however, interestingly, identical PFGE pattern was obtained in case of L265/2 and L850/2, although these strains derived from different nurseries (Figure 2). In conclusion, we could prove in both cases that the two $S$. aureus isolates carried by the same child were different from one another.

\section{Discussion}

Our results show that approximately one third of the Hungarian healthy preschool children are nasal $S$. aureus carriers. It is in good concordance with other studies' findings. For instance, Ciftci et al. [12] found $28.4 \%$ prevalence screening 1,134 4- to 6-year old healthy Turkish children. In Brazil, 31.1\% of 1,192 children attending DCCs carried S. aureus in their nose, as demonstrated by LamaroCardoso et al. [13]. However, in the literature, we can also find dissimilar

Table II. Comparison of the Staphylococcus aureus isolate pairs carried by the same child

\begin{tabular}{llcccccccc}
\hline & & \multicolumn{1}{c}{ MIC (mg/L) } \\
\cline { 3 - 9 } Isolate & Hemolysis type & \multirow{2}{*}{ PEN } & OXA & ERY & CLI & TET & GEN & MUP & CIP \\
\hline L265/1 & Delta & 0.25 & 0.25 & 0.5 & 0.125 & 0.25 & 0.5 & 0.064 & 0.25 \\
L265/2 & Alpha + beta + delta & 16 & 0.5 & 0.5 & 0.125 & 0.25 & 0.5 & 0.064 & 0.25 \\
L850/1 & Delta & 0.064 & 0.25 & 0.5 & 0.125 & 0.25 & 0.25 & 0.064 & 0.25 \\
L850/2 & Alpha + beta + delta & 2 & 0.25 & 0.5 & 0.125 & 0.25 & 0.25 & 0.064 & 0.5 \\
\hline
\end{tabular}

Note: PEN: penicillin; OXA: oxacillin; ERY: erythromycin; CLI: clindamycin; TET: tetracycline; GEN: gentamicin; MUP: mupirocin; CIP: ciprofloxacin; MIC: minimum inhibitory concentration. 


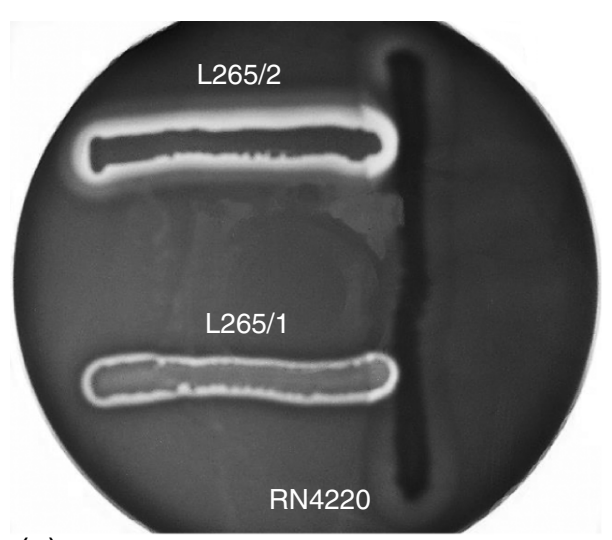

(a)

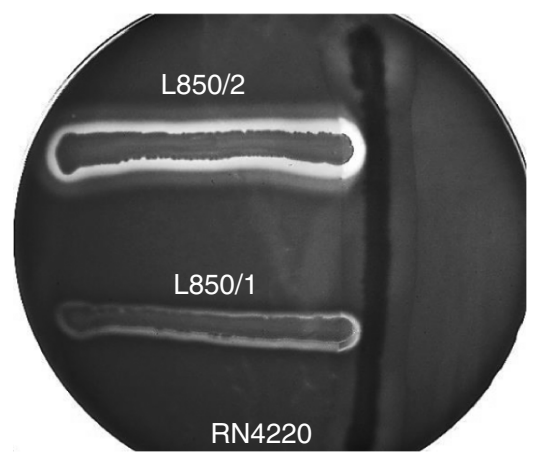

(b)

Figure 1. Determination of hemolytic phenotypes of the co-carried Staphylococcus aureus isolates, photo by K. Laub. (a) L265/1,2. (b) L850/1,2

prevalence data for $S$. aureus nasal colonization. For instance, Chatterjee et al. [14] presented much higher carriage rate $(52.3 \%)$ among Indian children. On the other hand, some studies observed lower colonization rates. Van Bijnen et al. published an ecological study in 2015, involving eight European countries (including Hungary), in which nearly 29,000 healthy persons aged $>4$ years were screened for nasal commensal $S$. aureus. Unfortunately no country-specific results were published, but the average carriage rate was $21.2 \%$ [15]. Similarly, in our previous study involving 878 healthy children from different cities of Hungary, we calculated a nearly identical carriage rate as $21.3 \%$ [5].

In the examined population, males exceeded females $(52.7 \%$ vs. $47.3 \%)$. Among the $S$. aureus carriers, this ratio was even higher $(58.2 \%$ 


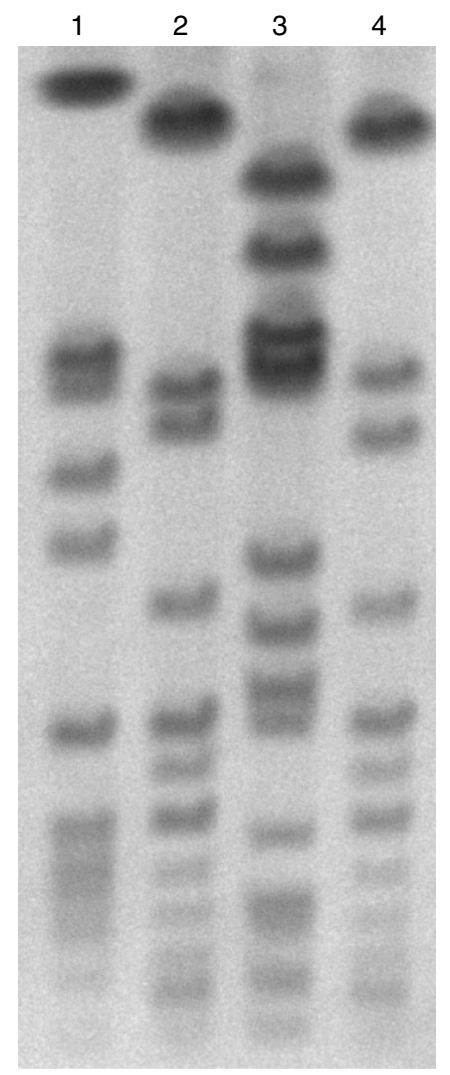

Figure 2. PFGE patterns of the two Staphylococcus aureus isolates each deriving from the same child. Lane 1, L265/1; lane 2, L265/2; lane 3, L850/1; lane 4, L850/2

vs. $41.8 \%)$. This difference proved to be statistically significant $(p=0.003)$. This finding is supported by Wertheim et al. [1], who concluded that $S$. aureus carriage rate is higher in men. In contrast, there are several examples where gender was not found to be a risk factor [16-18]. The other three possible risk factors - having siblings, siblings attending community, and passive smoking - did not correlate with nasal carriage in this study. Regev-Yochay et al. [19] concluded parental S. aureus colonization as an independent predictor for staphylococcal carriage in young children, but not having young siblings. Esposito et al. [17] did not find passive smoking a risk factor for carriage in children either. On the other hand, Melles et al. [20] revealed an association between passive smoking and carriage of a specific $S$. aureus genetic cluster in children. 
In this study, the antibiotic resistance rates were $<1 \%$ for oxacillin, gentamicin, ciprofloxacin, and mupirocin. Tetracycline resistance (R) was $3.4 \%$, whereas erythromycin and clindamycin $\mathrm{R}$ were almost the same $(10.3 \%$ and $9.5 \%$, respectively). Similar results were observed in a recent study conducted in Malaysia in the case of doxycycline $(4.2 \% \mathrm{R})$ and gentamicin $(0.0 \% \mathrm{R})$. Compared with our results, they measured higher resistance to ciprofloxacin (27.1\%) and erythromycin (20\%) [21]. In the study of van Bijnen et al. [15], penicillin resistance among the 539 Hungarian isolates was $76.1 \%$ and considering all the eight countries, it varied between $64.8 \%$ and $87.1 \%$; erythromycin $\mathrm{R}$ was $12.1 \%$ (range: $1.5 \%-16.5 \%$ ); clindamycin $\mathrm{R}$ was $12.1 \%$ (range: $1.4 \%-14.6 \%$ ); tetracycline $\mathrm{R}$ was $6.7 \%$ (range: $1.8 \%-7.2 \%$ ); gentamicin $\mathrm{R}$ was $0.0 \%$ (range: $0.0 \%-2.0 \%$ ); and ciprofloxacin $\mathrm{R}$ was $2.0 \%$ (range: $0.6 \%-2.5 \%$ ). We can conclude that among children, tetracycline resistance is lower, whereas to erythromycin, clindamycin, gentamicin, and ciprofloxacin are similar to those in the aforementioned paper. We were interested in the mupirocin sensitivity as well, because it is used for MRSA nasal decolonization [22]. Fortunately, all our isolates (including MRSAs) were susceptible to it. In Hungary, the National Center for Epidemiology (NCE) annually reports antibiotic sensitivity data separated to inpatients and outpatients [23]. We analyzed these data in case of $S$. aureus in 2012 and compared with our results as shown in Figure 3.

To conclude, carried isolates are more sensitive to the tested antibiotics than clinical ones except for penicillin.

All four MRSA isolates in this study were resistant to erythromycin. NCE data show approximately $84 \%$ erythromycin resistance among HA-MRSA strains [23].

The NCE database has antibiotic resistance data not only for $S$. aureus but for clinical MRSA isolates (HA-MRSA) as well. In this study, the CA-MRSA rate among healthy children carrying $S$. aureus was $0.8 \%$, whereas this rate in outpatients in Hungary was $7.8 \%$ in the same year. Among inpatients, MRSA prevalence is generally higher than among outpatients, according to NCE, it was $23.0 \%$ in 2012 [23]. In a recent Serbian study, Lepsanovic et al. [24] found 3.8\% MRSA prevalence among 1,362 healthy adult carriers, while $45.8 \%$ of $S$. aureus isolates collected from hospitalized patients proved to be MRSA. In our previous study, 878 healthy children were screened all over Hungary, of which 187 carried S. aureus and none of them were methicillin-resistant [5]. If we now combine these results with this study, we get $29.1 \%$ overall carriage rate and $0.6 \%$ cumulated MRSA prevalence rate among $S$. aureus isolates. We can establish that CA-MRSA carriage rate is very low in Hungary in small children. Van Bijnen et al. [15] found a similarly low prevalence of MRSA (1.3\%) among 6,093 S. aureus isolates in Europe in the aforementioned study. Regev-Yochay et al. [19] demonstrated a 


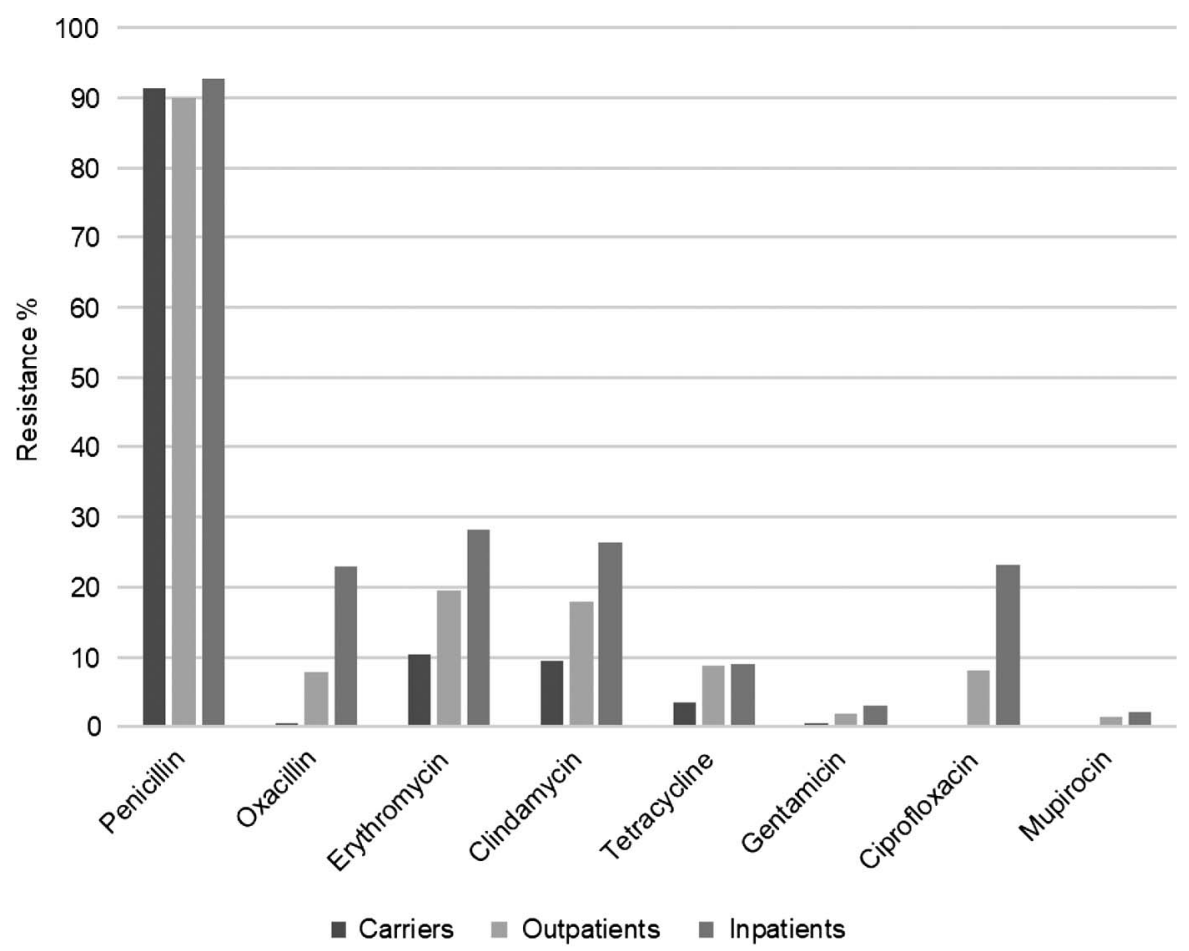

Figure 3. Antibiotic resistance rates of carried Staphylococcus aureus in this study $(n=476)$ versus clinical isolates (nationwide data obtained from the National Center for Epidemiology, $n>5,000$ ) from Hungary in 2012

similar CA-MRSA carriage rate (less than $0.1 \%$ ) examining more than 4,500 children in Israel. In an Iranian study, 5.8\% of 2- to 6-year-old children were colonized with CA-MRSA [16].

All four mecA positive isolates of this study were of SCCmec type IV. In most of the world, SCCmec types IV and $\mathrm{V}$ are most prevalent among CA-MRSA strains; however, mostly clinical isolates are investigated. For example, in the aforementioned recent Serbian study, 40 isolates out of the total 50 investigated belonged to these two types [24]. Nonetheless, best comparison could be done with similar studies, involving $S$. aureus isolated from healthy children. In Jordan, Alzoubi et al. [25] collected nasal specimens from children aged 6-11 years and all 15 MRSA identified were type IV. Another very similar project to ours was conducted in Argentina, where 316 healthy children were screened in a given city, and here again all 14 MRSA strains carried the SCCmec type IV cassette [26]. On the other hand, in India, where 
1,503 school-age children were screened, only 7 out of the 17 MRSAs belonged to type IV and none were identified as type V either [27]. The MLST typing revealed that all MRSA isolates in this study belonged to ST45, a clone which has been very frequently reported from numerous countries from all over the world, based on the MLST database [9].

\section{Acknowledgements}

The authors would like to thank Prof. Richard Novick for providing RN4220 for the hemolysis test. This work was financially supported by the National Research, Development and Innovation Office/Hungarian Scientific Research Fund (grant number K108631).

\section{Conflict of Interest}

The authors declare no conflict of interest.

\section{References}

1. Wertheim, H. F., Melles, D. C., Vos, M. C., van Leeuwen, W., van Belkum, A., Verbrugh, H. A., Nouwen, J. L.: The role of nasal carriage in Staphylococcus aureus infections. Lancet Infect Dis 5, 751-762 (2005).

2. Barber, M.: Methicillin-resistant staphylococci. J Clin Pathol 14, 385-393 (1961).

3. Rehm, S. J., Tice, A.: Staphylococcus aureus: Methicillin-susceptible S. aureus to methicillin-resistant $S$. aureus and vancomycin-resistant $S$. aureus. Clin Infect Dis 51, S176-S182 (2010).

4. Cooke, F. J., Brown, N. M.: Community-associated methicillin-resistant Staphylococcus aureus infections. Br Med Bull 94, 215-227 (2010).

5. Laub, K., Tóthpál, A., Kardos, S., Dobay, O.: Epidemiology and antibiotic sensitivity of Staphylococcus aureus nasal carriage in children in Hungary. Acta Microbiol Immunol Hung 64, 51-62 (2017).

6. Paterson, G. K., Larsen, A. R., Robb, A., Edwards, G. E., Pennycott, T. W., Foster, G., Mot, D., Hermans, K., Baert, K., Peacock, S. J., Parkhill, J., Zadoks, R. N., Holmes, M. A.:

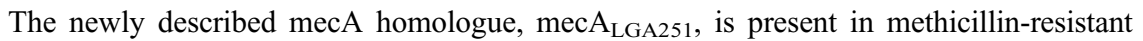
Staphylococcus aureus isolates from a diverse range of host species. J Antimicrob Chemother 67, 2809-2813 (2012).

7. Milheiriço, C., Oliveira, D. C., de Lencastre, H.: Update to the multiplex PCR strategy for assignment of mec element types in Staphylococcus aureus. Antimicrob Agents Chemother 51, 3374-3377 (2007).

8. Oliveira, D. C., de Lencastre, H.: Multiplex PCR strategy for rapid identification of structural types and variants of the mec element in methicillin-resistant Staphylococcus aureus. Antimicrob Agents Chemother 46, 2155-2161 (2002). 
9. Multi Locus Sequence Typing (MLST) website. Available at http://saureus.mlst.net/.

10. The European Committee on Antimicrobial Susceptibility Testing (EUCAST): Breakpoint tables for interpretation of MICs and zone diameters. Version 7.1 (2017). Available at http://www.eucast.org/clinical_breakpoints/.

11. Herbert, S., Ziebandt, A. K., Ohlsen, K., Schäfer, T., Hecker, M., Albrecht, D., Novick, R., Götz, F.: Repair of global regulators in Staphylococcus aureus 8325 and comparative analysis with other clinical isolates. Infect Immun 78, 2877-2889 (2010).

12. Ciftci, I. H., Koken, R., Bukulmez, A., Ozdemir, M., Safak, B., Cetinkaya, Z.: Nasal carriage of Staphylococcus aureus in 4-6 age groups in healthy children in Afyonkarahisar, Turkey. Acta Paediatr 96, 1043-1046 (2007).

13. Lamaro-Cardoso, J., de Lencastre, H., Kipnis, A., Pimenta, F. C., Oliveira, L. S., Oliveira, R. M., Nouer, S. S., Aires-de-Sousa, M., Milheiriço, C., Andrade, A. L.: Molecular epidemiology and risk factors for nasal carriage of Staphylococcus aureus and methicillin-resistant $S$. aureus in infants attending day care centers in Brazil. J Clin Microbiol 47, 3991-3997 (2009).

14. Chatterjee, S. S., Ray, P., Aggarwal, A., Das, A., Sharma, M.: A community-based study on nasal carriage of Staphylococcus aureus. Indian J Med Res 130, 742-748 (2009).

15. van Bijnen, E. M., Paget, J., de Lange-de Klerk, E. S., den Heijer, C. D., Versporten, A., Stobberingh, E. E., Goossens, H., Schellevis, F. G., APRES Study Team: Antibiotic exposure and other risk factors for antimicrobial resistance in nasal commensal Staphylococcus aureus: An ecological study in 8 European countries. PLoS One 10, e0135094 (2015).

16. Davoodabadi, F., Mobasherizadeh, S., Mostafavizadeh, K., Shojaei, H., Havaei, S. A., Koushki, A. M., Moghadasizadeh, Z., Meidani, M., Shirani, K.: Nasal colonization in children with community acquired methicillin-resistant Staphylococcus aureus. Adv Biomed Res 5, 86 (2016).

17. Esposito, S., Terranova, L., Zampiero, A., Ierardi, V., Rios, W. P., Pelucchi, C., Principi, N.: Oropharyngeal and nasal Staphylococcus aureus carriage by healthy children. BMC Infect Dis 14, 723 (2014).

18. Van Nguyen, K., Zhang, T., Thi Vu, B. N., Dao, T. T., Tran, T. K., Thi Nguyen, D. N., Thi Tran, H. K., Thi Nguyen, C. K., Fox, A., Horby, P., Wertheim, H.: Staphylococcus aureus nasopharyngeal carriage in rural and urban northern Vietnam. Trans R Soc Trop Med Hyg 108, 783-790 (2014).

19. Regev-Yochay, G., Raz, M., Carmeli, Y., Shainberg, B., Navon-Venezia, S., Pinco, E., Leavitt, A., Keller, N., Rahav, G., Malley, R., Rubinstein, E., Maccabi Implementing Judicious Antibiotic Prescription Study Group: Parental Staphylococcus aureus carriage is associated with staphylococcal carriage in young children. Pediatr Infect Dis J 28, 960-965 (2009).

20. Melles, D. C., Bogaert, D., Gorkink, R. F., Peeters, J. K., Moorhouse, M. J., Ott, A., van Leeuwen, W. B., Simons, G., Verbrugh, H. A., Hermans, P. W., van Belkum, A.: Nasopharyngeal co-colonization with Staphylococcus aureus and Streptococcus pneumoniae in children is bacterial genotype independent. Microbiology 153, 686-692 (2007).

21. Mohamed, N. A., Ramli, S., Amin, N. N., Sulaiman, W. S., Isahak, I., Jamaluddin, T. Z., Salleh, N. M.: Staphylococcus aureus carriage in selected kindergartens in Klang Valley. Med J Malaysia 71, 62-65 (2016). 
22. Ammerlaan, H. S., Kluytmans, J. A., Wertheim, H. F., Nouwen, J. L., Bonten, M. J.: Eradication of methicillin-resistant Staphylococcus aureus carriage: A systematic review. Clin Infect Dis 48, 922-930 (2009).

23. National Bacteriological Surveillance Management Team: NBS Annual reports. National Center for Epidemiology, Budapest, Hungary. Available at http://www.oek.hu/oek.web.

24. Lepsanovic, Z., Jeremic, L. P., Lazic, S., Cirkovic, I.: High prevalence and resistance patterns of community-associated methicillin-resistant Staphylococcus aureus in the Pomoravlje Region, Serbia. Acta Microbiol Immunol Hung 63, 83-92 (2016).

25. Alzoubi, H. M., Aqel, A. A., Al-Sarayreh, S. A., Al-Zayadneh, E.: Methicillin-resistant Staphylococcus aureus nasal carriage among primary school-aged children from Jordan: Prevalence, antibiotic resistance and molecular characteristics. J Egypt Public Health Assoc 89, 114-118 (2014).

26. Gardella, N., Murzicato, S., Di Gregorio, S., Cuirolo, A., Desse, J., Crudo, F., Gutkind, G., Mollerach, M.: Prevalence and characterization of methicillin-resistant Staphylococcus aureus among healthy children in a city of Argentina. Infect Genet Evol 11, 1066-1071 (2011).

27. Govindan, S., Maroli, A. S., Ciraj, A. M., Bairy, I.: Molecular epidemiology of methicillin resistant Staphylococcus aureus colonizing the anterior nares of school children of Udupi Taluk. Indian J Med Microbiol 33, 129-133 (2015). 